\title{
Transient response of a finite crack subjected to dynamic anti-plane loading
}

\author{
YI-SHYONG ING and CHIEN-CHING MA \\ Department of Mechanical Engineering, National Taiwan University, Taipei, Taiwan 10617, Republic of China
}

Received 20 May 1996; accepted in revised form 21 October 1996

\begin{abstract}
In this study, the transient response of a finite crack in an elastic solid subjected to dynamic antiplane loading is investigated. Two specific loading situations, a body force near the finite crack and a concentrated point loading applied on the crack face, are analyzed in detail. In analyzing this problem, an infinite number of diffracted waves generated by two crack tips must be taken into account which will make the analysis extremely difficult. The solutions are determined by superposition of proposed fundamental solutions in the Laplace transform domain. The fundamental solutions to be used are the problems for applying exponentially distributed traction and screw dislocation to the crack faces and along the crack-tip line respectively. Exact transient closed-form solutions for the dynamic stress intensity factor are obtained and expressed in very simple and compact formulations. The solutions are valid for an infinite length of time and have accounted for the contributions of an infinite number of diffracted waves. Numerical calculations for the two problems are evaluated and results indicate that the dynamic stress intensity factors will oscillate near the corresponding static values after the first three waves have passed through the specified crack tip.
\end{abstract}

\section{Introduction}

Recently, the transient response of a solid medium containing a crack-like flaw under dynamic loads has received much attention. Most of the research, however, has been directed to the solution of problems with a semi-infinite crack subjected to symmetrically distributed impact loading on crack faces. The complete solutions mentioned above can be obtained by integral transform methods in conjunction with direct application of the Wiener-Hopf technique (Noble [1]) and the Cagniard-de Hoop method (de Hoop [2]) of Laplace inversion. If the cracked problem has a characteristic length or the loading condition is unsymmetrical, then the same procedure using integral transform methods does not apply.

The stress intensities at the edges of a finite crack subjected to the time-harmonic, horizontally polarized plane wave have been obtained by Loeber and Sih [3] and Sih and Loeber [4]. Thau and Lu [5], following the work of Kostrov [6] and Flitman [7], treated the analogous transient problem of diffraction of an arbitrary plane dilatational wave by a finite crack in an infinite elastic solid. Their results are exact only at the time interval in which the dilatational wave has traveled the length of the crack twice. Sih and Embley [8] have studied the near-field solution to the problem of a finite crack under transient in-plane loading. They reduced the mixed boundary value problem to a standard Fredholm integral equation and subsequently inverted the Laplace transform of the stress components by a combination of numerical means and application of the Cagniard inversion technique. A class of problems involving interaction between a finite crack and other boundaries was considered by Chen [9-10] and Itou [11-12]. With the exception of Loeber and Sih who considered the time-harmonic incident wave, all of the authors mentioned above have simplified their problems by assuming the symmetrically distributed loading conditions, and finally used a numerical Laplace inversion technique to 
obtain the solutions in the physical domain. Because of the mathematical difficulties, the closed form analytical solution to the problem of a finite crack loaded by an arbitrary located point loading has not yet been obtained.

The problem of an unbounded medium containing a semi-infinite crack subjected to a pair of concentrated loadings on the faces of the crack has been investigated by Freund [13]. He proposed a fundamental solution, arising from an edge dislocation climbing along the line ahead of the crack tip with a constant speed, to overcome these difficulties of the case with a characteristic length. The solution can be constructed by taking an integration over a climbing dislocation of different moving velocity. Basing his procedure on this method, Brock [14-16], and $\mathrm{Ma}$ and Hou [17-18] have analyzed a series of problems of a semi-infinite crack subjected to impact loading. A thorough summary of the application of the main direct methods of analysis for transient problem in dynamic fracture for elastic or inelastic problems has been given by Freund [19]. Freund [19] has suggested an alternate approach based on the aforementioned moving dislocation solution to examine the same finite-crack problem which had been solved by Thau and $\mathrm{Lu}$ [5]. In practice, however, the alternate approach provides a solution which is valid for the same time range as before.

Kostrov [20] and Achenbach [21-22] have used the method based on Green's function to solve the problems of crack propagation for anti-plane deformation. In their studies, the region of integration for the integral equation is in a complicated shape, generally being bounded by a hyperbola and a number of straight lines. For points ahead of the crack tip, the region of integration reduces to a triangular region and the stress in the plane of the crack can thus be determined without difficulty. However, for material points which are not on the crack-tip line, the region of integration is very complicated and careful analysis is needed. Brock [23] also investigated the similar problem for the long time behavior. His results indicated that the peak of dynamic stress intensity factors could occur after the arrival of the second diffracted wave, which means that secondary diffractions may produce even higher peaks than the earlier peaking. This result is different from that obtained in [21]. Whenever dynamic loading is applied to a body with an internal crack, the resulting stress waves may cause crack growth. Few solutions for a cracked elastic solid subjected to dynamic loading are available. Exact transient closed-form solutions for a stationary semi-infinite crack subjected to a suddenly applied dynamic body force in an unbounded medium have been obtained by Tsai and Ma [24] for the in-plane case and by Ma and Chen [25] for the anti-plane case. They determined the solutions by superimposing a fundamental solution in the Laplace transform domain. The fundamental solution used in the problem is an exponentially distributed traction in the Laplace transform domain applied on the crack faces. This fundamental solution has also been applied successfully to solve the problems of a half plane containing a semi-infinite inclined crack by Tsai and $\mathrm{Ma}$ [26] and $\mathrm{Ma}$ and Chen [27] for in-plane and anti-plane problems, respectively.

In this study, the transient response of a finite crack in an unbounded medium subjected to dynamic anti-plane point loading is investigated. Two specific loading conditions, a dynamic concentrated body force (Figure 1) and a concentrated point loading applied on the crack face (Figure 8), are considered in detail. In analyzing this problem, the interaction of two crack tips must be taken into account which will make the analysis extremely difficult. It is impossible to solve this complicated problem by using the standard Wiener-Hopf technique and other approaches must be followed. Two useful fundamental solutions are proposed to overcome these difficulties. The proposed fundamental solutions are successfully applied towards solving the problems and are demonstrated as an efficient methodology to solve 


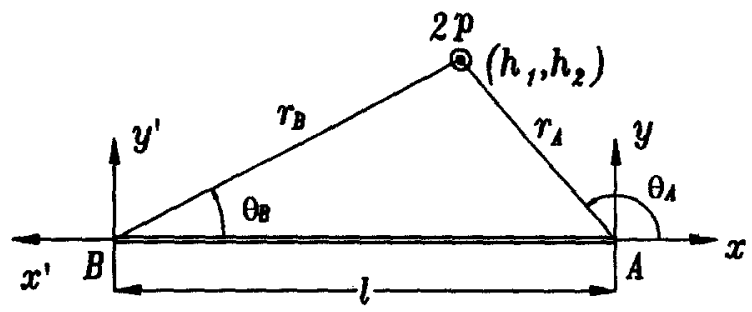

Figure 1. Configuration and coordinate systems of a finite crack subjected to dynamic body forces.

similar problems. A compact and explicit closed-form solution is obtained and every term expressed in the solution has its own physical meaning. Since the stress intensity factor is the key parameter in characterizing dynamic crack growth, we will focus our attention mainly on the determination of the dynamic stress intensity factor.

\section{Required fundamental solutions}

In this paper, two alternative fundamental solutions are proposed and successfully applied to solve the aforementioned problems of a finite crack subjected to dynamic loadings. The solutions for an exponentially distributed traction applied to the crack faces, and an exponentially, distributed screw dislocation along the crack tip in the Laplace transform domain will be referred to as the fundamental solutions. The diffracted waves generated from the crack tips can be constructed by superimposing the fundamental solutions in the Laplace transform domain.

The governing equation for the anti-plane problem is represented by the two-dimensional wave equation

$$
\frac{\partial^{2} w}{\partial x^{2}}+\frac{\partial^{2} w}{\partial y^{2}}=b^{2} \frac{\partial^{2} w}{\partial t^{2}},
$$

where $w$ is the out-of-plane displacement, and $b$ is the slowness of the shear wave given by

$$
b=\frac{1}{\nu}=\sqrt{\frac{\rho}{\mu}} .
$$

Here $\nu$ is the shear wave speed, $\mu$ and $\rho$ are the respective shear modulus and the mass density of the material. The non-vanishing shear stresses are

$$
\tau_{y z}=\mu \frac{\partial w}{\partial y}, \quad \tau_{x z}=\mu \frac{\partial w}{\partial x} .
$$

(1) Fundamental solution for distributed loads on crack faces

An anti-plane exponentially distributed traction in the Laplace transform domain is applied to the upper and lower crack faces. Because the tractions are equal but opposite on the two crack faces, the problem can be viewed as a half-plane problem with the material occupying the region $y \geqslant 0$, and subjected to the following mixed boundary conditions in the Laplace transform domain

$$
\bar{\tau}_{y z}(x, 0, s)=\mathrm{e}^{s \eta x}, \quad \text { for }-\infty<x<0,
$$




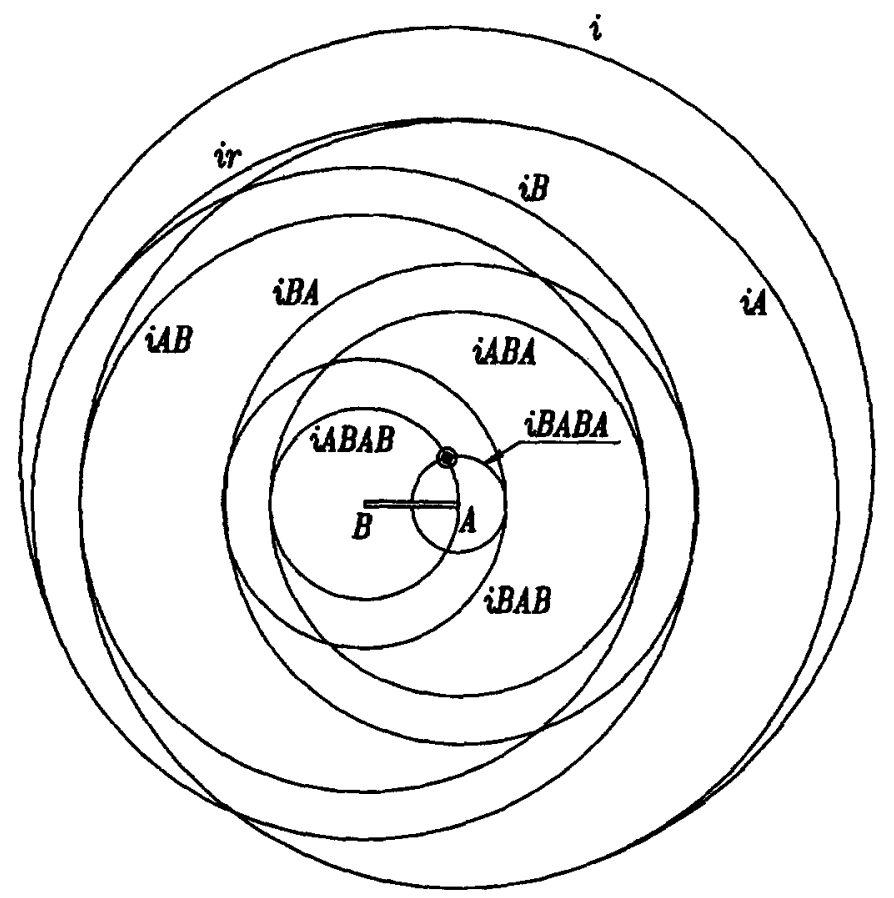

Figure 2. Wave fronts of the incident and diffracted waves for a short time period after applying dynamic body forces.

$$
\bar{w}(x, 0, s)=0, \quad \text { for } 0<x<\infty,
$$

where $s$ is the Laplace transform parameter and $\eta$ is a constant. The overbar symbol is used for denoting the transform on time $t$. This fundamental problem can be solved by the application of integral transforms. Applying the one-sided Laplace transform over time, and the two-sided Laplace transform over $x$, under the restriction of $\operatorname{Re}(\eta)>\operatorname{Re}(\lambda)$, the Wiener-Hopf technique is finally implemented. The solutions for stresses and displacement in the Laplace transform domain, which satisfy the boundary conditions (3) and (4), can be expressed as follows

$$
\begin{aligned}
& \bar{\tau}_{y z}(x, y, s)=\frac{1}{2 \pi i} \int_{\Gamma_{\lambda}} \frac{(b+\lambda)^{1 / 2} \mathrm{e}^{-s(\alpha y-\lambda x)}}{(b+\eta)^{1 / 2}(\eta-\lambda)} \mathrm{d} \lambda, \\
& \bar{\tau}_{x z}(x, y, s)=\frac{-1}{2 \pi i} \int_{\Gamma_{\lambda}} \frac{\lambda \mathrm{e}^{-s(\alpha y-\lambda x)}}{(b+\eta)^{1 / 2}(\eta-\lambda)(b-\lambda)^{1 / 2}} \mathrm{~d} \lambda, \\
& \bar{w}(x, y, s)=\frac{-1}{2 \pi i} \int_{\Gamma_{\lambda}} \frac{\mathrm{e}^{-s(\alpha y-\lambda x)}}{\mu s(b+\eta)^{1 / 2}(\eta-\lambda)(b-\lambda)^{1 / 2}} \mathrm{~d} \lambda .
\end{aligned}
$$

The corresponding result of the dynamic stress intensity factor in the Laplace transform domain is

$$
\begin{aligned}
\bar{K}(s) & =\lim _{x \rightarrow 0} \sqrt{2 \pi x} \bar{\tau}_{y z}(x, 0, s) \\
& =-\frac{\sqrt{2}}{\sqrt{s}(b+\eta)^{1 / 2}} .
\end{aligned}
$$




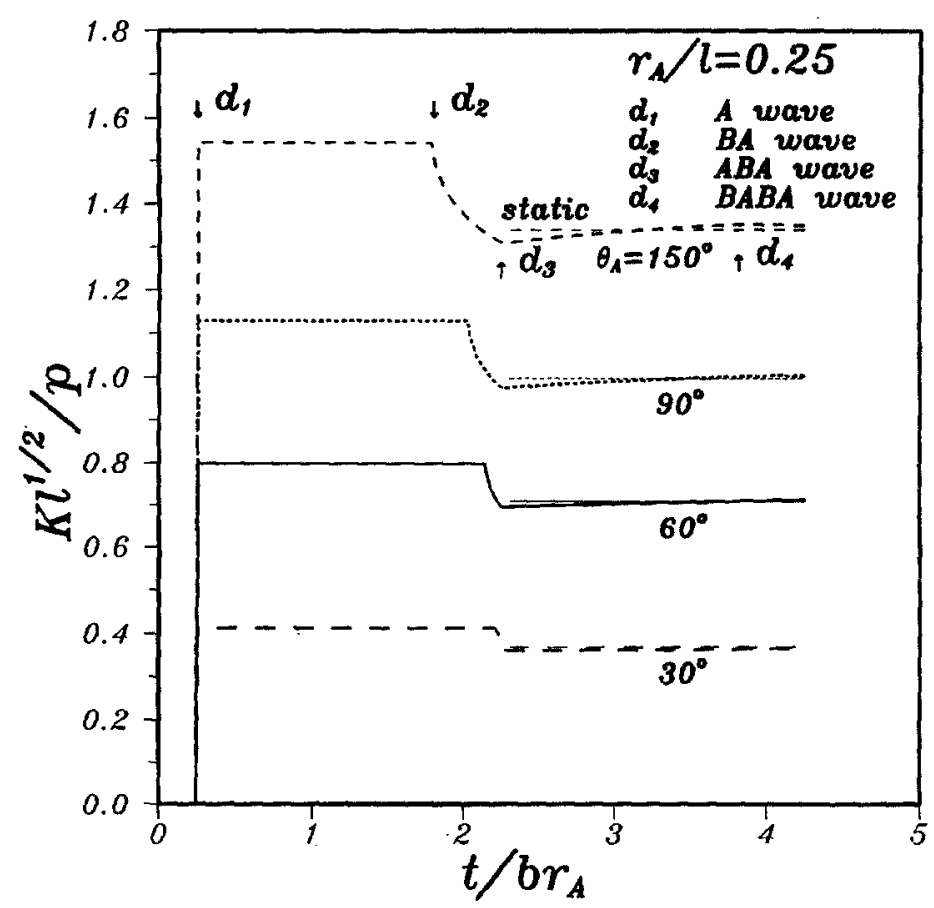

Figure 3. Stress intensity factors for applying dynamic body forces at $r_{A}=l / 4$ for different values of $\theta_{A}$.

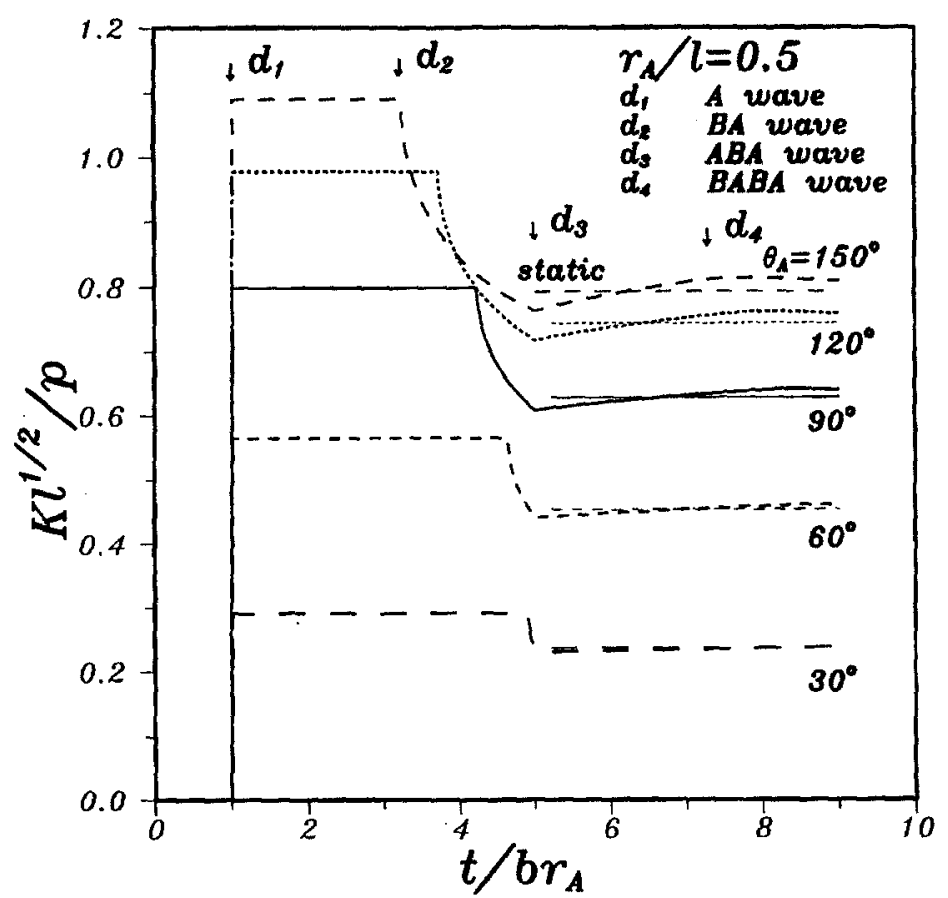

Figure 4. Stress intensity factors for applying dynamic body forces at $r_{A}=l / 2$ for different values of $\theta_{A}$. 


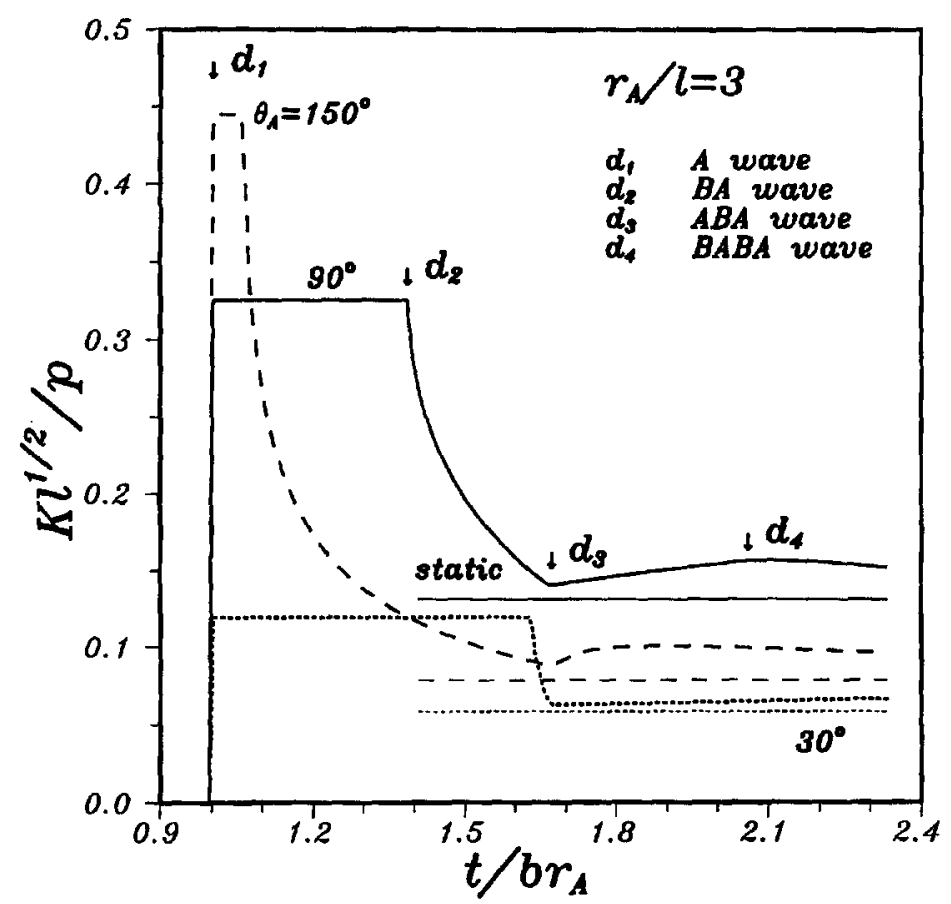

Figure 5. Stress intensity factors for applying dynamic body forces at $r_{A}=3 l$ for different values of $\theta_{A}$.

(2) Fundamental solution of screw dislocation ahead of the crack tip

Consider a semi-infinite crack contained in an unbounded medium. A distributed screw dislocation ahead of the crack tip yields the following boundary conditions in the Laplace transform domain

$$
\begin{aligned}
& \bar{w}(x, 0, s)=\mathrm{e}^{s \eta x}, \quad \text { for } 0<x<\infty, \\
& \bar{\tau}_{y z}(x, 0, s)=0, \text { for }-\infty<x<0 .
\end{aligned}
$$

The solutions for stresses and displacement expressed in the Laplace transform domain are

$$
\begin{aligned}
& \bar{\tau}_{y z}(x, y, s)=\frac{1}{2 \pi i} \int_{\Gamma_{\lambda}} \frac{\mu s(b-\eta)^{1 / 2}(b+\lambda)^{1 / 2} \mathrm{e}^{-s(\alpha y-\lambda x)}}{(\eta-\lambda)} \mathrm{d} \lambda \\
& \bar{\tau}_{x z}(x, y, s)=\frac{-1}{2 \pi i} \int_{\Gamma_{\lambda}} \frac{\mu s(b-\eta)^{1 / 2} \mathrm{e}^{-s(\alpha y-\lambda x)}}{(\eta-\lambda)(b-\lambda)^{1 / 2}} \mathrm{~d} \lambda \\
& \bar{w}(x, y, s)=\frac{-1}{2 \pi i} \int_{\Gamma_{\lambda}} \frac{(b-\eta)^{1 / 2} \mathrm{e}^{-s(\alpha y-\lambda x)}}{(\eta-\lambda)(b-\lambda)^{1 / 2}} \mathrm{~d} \lambda .
\end{aligned}
$$

The corresponding result of the stress intensity factor expressed in the Laplace transform domain is

$$
\bar{K}(s)=-\mu \sqrt{2 s}(b-\eta)^{1 / 2} .
$$




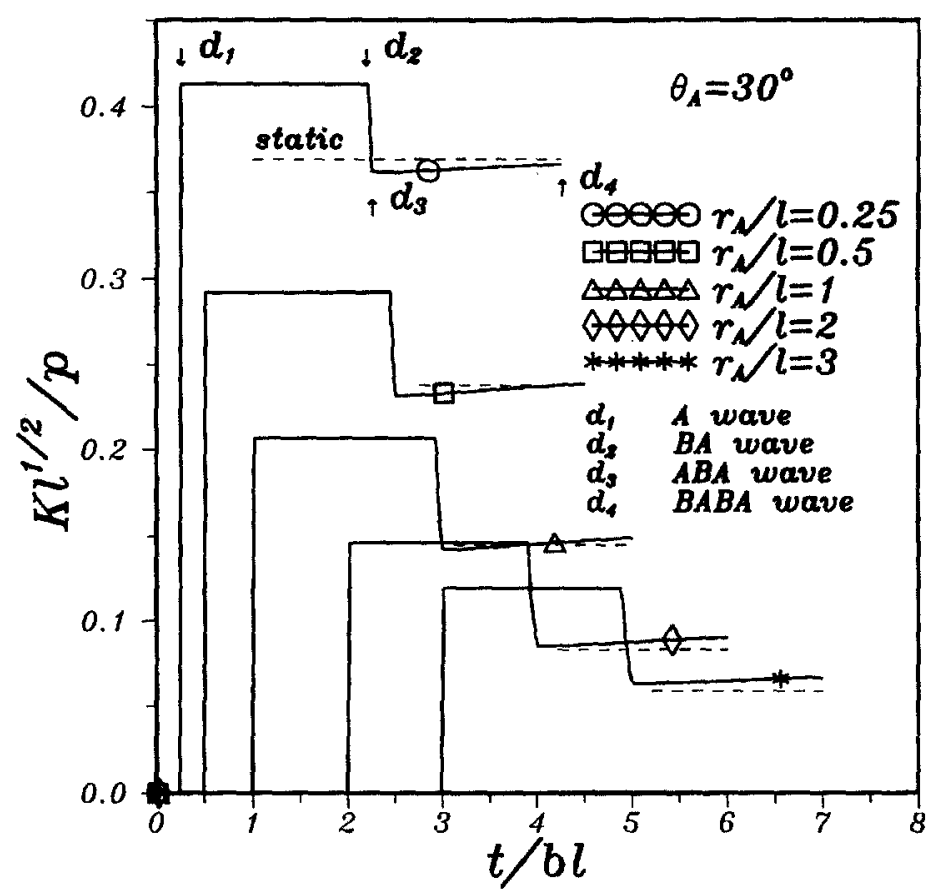

Figure 6. Stress intensity factors for applying dynamic body forces at $\theta_{A}=30^{\circ}$ for different values of $r_{A}$.

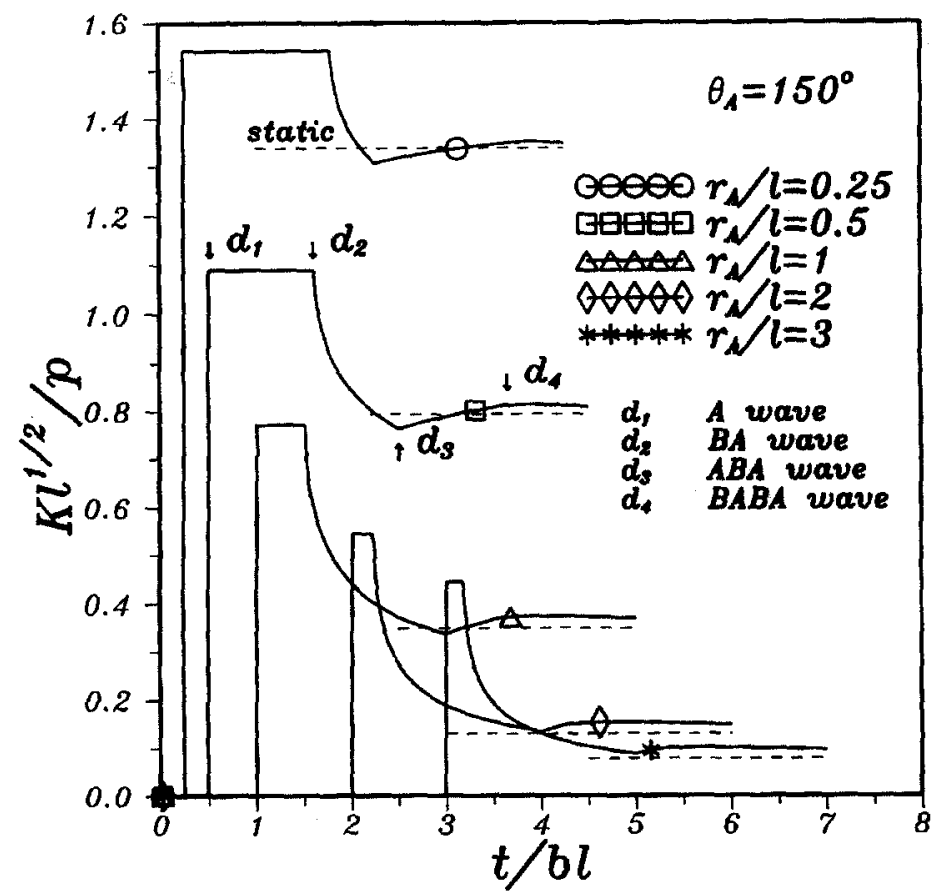

Figure 7. Stress intensity factors for applying dynamic body forces at $\theta_{A}=150^{\circ}$ for different values of $r_{A}$. 


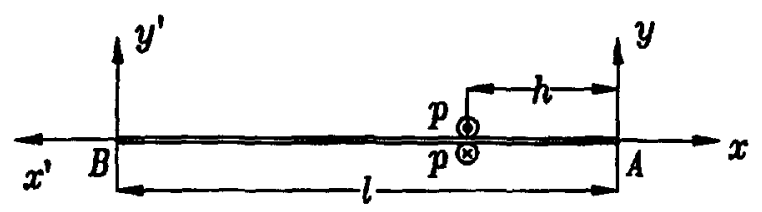

Figure 8. Configuration and coordinate systems of a finite crack subjected to dynamic anti-plane shear forces on the crack faces.

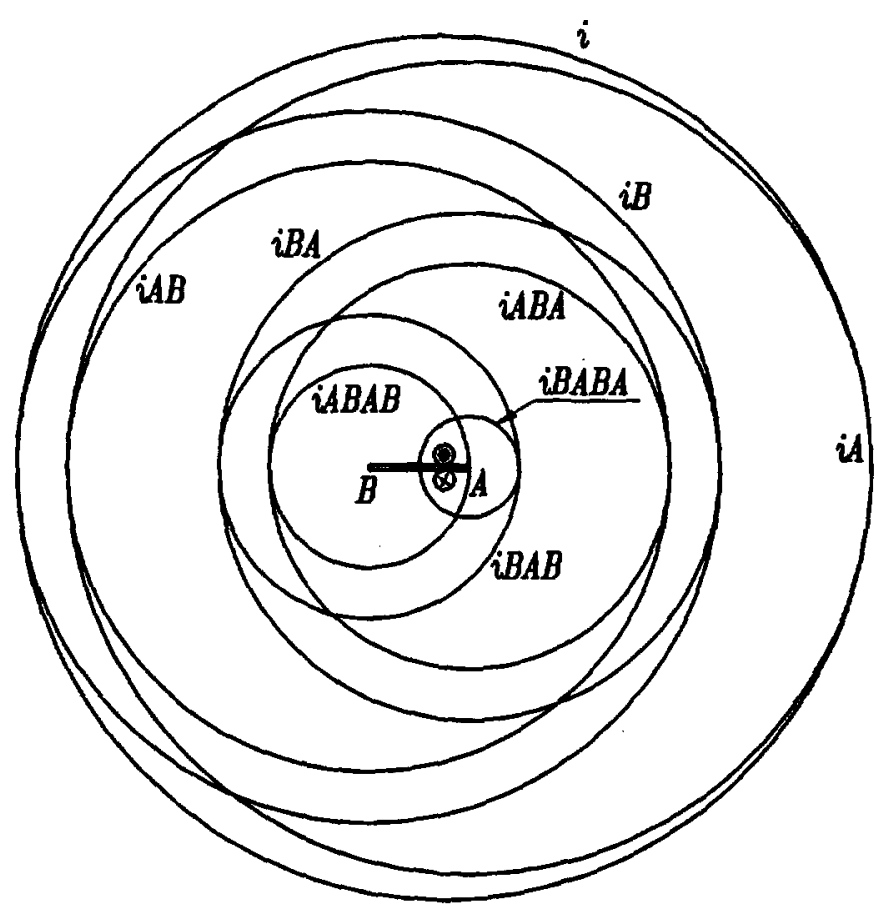

Figure 9. Wave fronts of the incident and diffracted waves for a short time period after applying dynamic anti-plane shear forces on the crack faces.

\section{Dynamic stress intensity factors for applying dynamic body forces}

A specific geometry to be considered here is an infinite medium containing a finite crack of length $l$ as shown in Figure 1. The origins of two coordinate systems $(x, y)$ and $\left(x^{\prime}, y^{\prime}\right)$ are located at crack tips $A$ and $B$, respectively. For $t<0$, the medium is stress free and at rest. At time $t=0$, a dynamic concentrated anti-plane body force acts at $x=h_{1}, y=h_{2}$. After applying the dynamic impact, the incident cylindrical stress wave generated from the loading point will arrive at the crack tips $A$ and $B$, and diffracted waves are induced from crack tips. The diffracted waves will scatter back and forth between the crack tips $A$ and $B$ at a later time. In analyzing this problem, the diffractions of stress waves by the finite crack, which will generate an infinite number of waves, must be taken into account.

An effective superposition scheme will be proposed in this study to solve the complicated problem. No loss of generality, we focus our attention on diffracted waves which are induced by the crack tip $A$. The dynamic concentrated body force is represented by

$$
\tau_{y z}\left(x, h_{2}^{+}, t\right)-\tau_{y z}\left(x, h_{2}^{-}, t\right)=2 p \delta\left(x-h_{1}\right) \mathrm{H}(t) .
$$




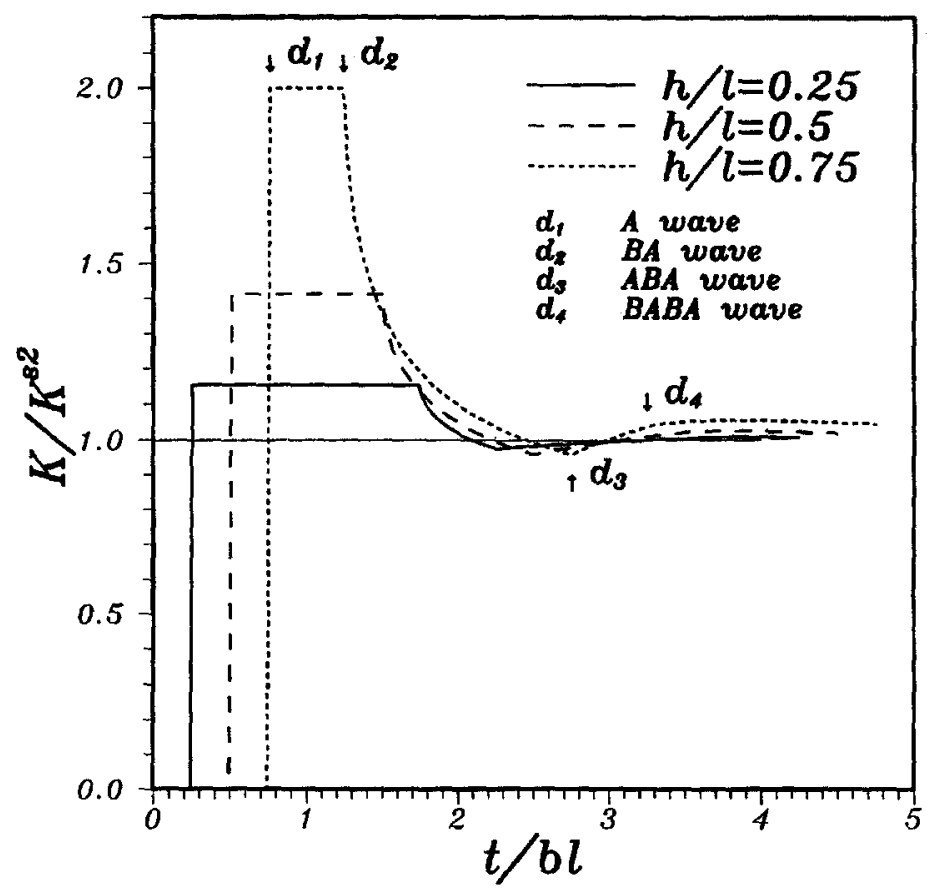

Figure 10. Stress intensity factors for applying dynamic anti-plane shear forces on the crack faces for $h / l=$ $0.25,0.5$ and 0.75 .

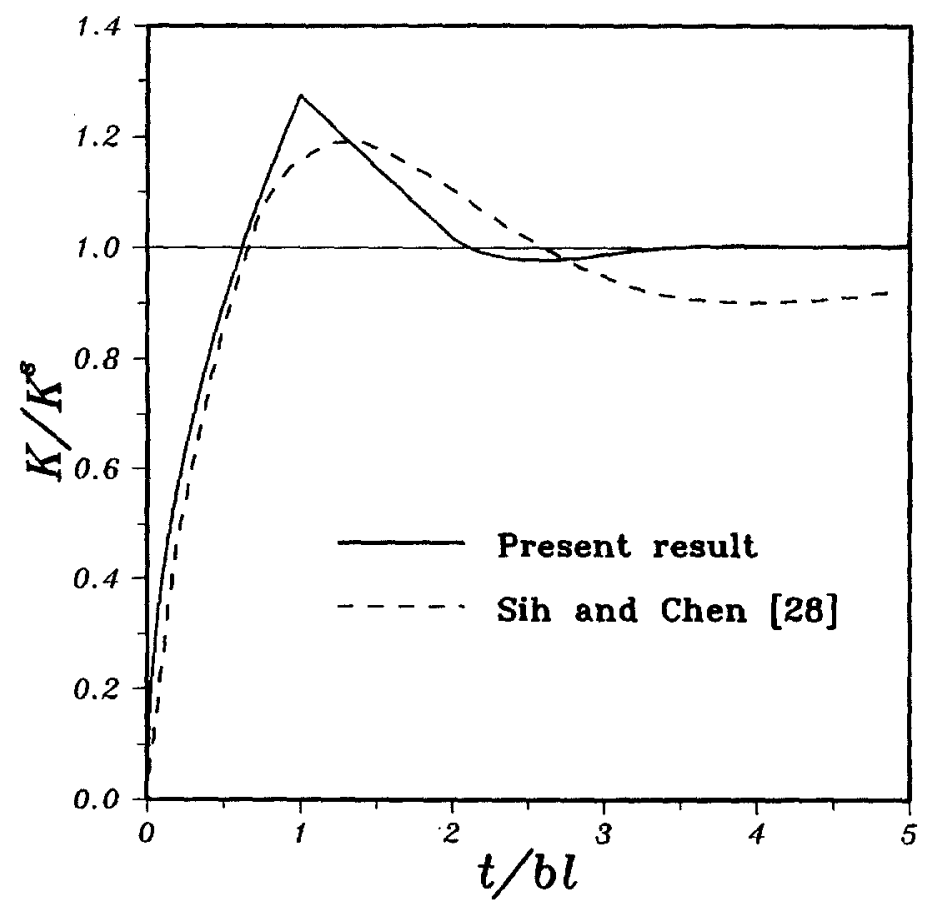

Figure 11. Stress intensity factors for applying anti-plane uniformly distributed dynamic loading on the crack faces. 
The incident field for $y \leqslant h_{2}$ in the Laplace transform domain can be represented as

$$
\bar{\tau}_{y z}^{i}(x, y, s)=\frac{1}{2 \pi i} \int_{\Gamma_{\lambda}} p \mathrm{e}^{s \alpha\left(y-h_{2}\right)+s \lambda\left(x-h_{1}\right)} \mathrm{d} \lambda,
$$

or expressed in the $\left(x^{\prime}, y^{\prime}\right)$ coordinate system as

$$
\bar{\tau}_{y^{\prime} z^{\prime}}^{i}\left(x^{\prime}, y^{\prime}, s\right)=\frac{-1}{2 \pi i} \int_{\Gamma_{\lambda}} p \mathrm{e}^{s \alpha\left(y^{\prime}-h_{2}\right)+s \lambda\left[x^{\prime}+\left(l+h_{1}\right)\right]} \mathrm{d} \lambda,
$$

where

$$
\alpha(\lambda)=\alpha_{+}(\lambda) \alpha_{-}(\lambda)=(b+\lambda)^{1 / 2}(b-\lambda)^{1 / 2} .
$$

Before the stress wave is diffracted from the crack tip $B$, the stress field is precisely the same as that derived for a semi-infinite crack which lies in the plane $y=0$ and $-\infty<x<0$ and subjected to the same loading. The incident stress field $\bar{\tau}_{y z}^{i}(x, 0, s)$ at $y=0$ generated by the applied dynamic loading expressed in the Laplace transform domain is

$$
\bar{\tau}_{y z}^{i}(x, 0, s)=\frac{1}{2 \pi i} \int_{\Gamma_{\lambda}} p \mathrm{e}^{-s \alpha h_{2}+s \lambda\left(x-h_{1}\right)} \mathrm{d} \lambda .
$$

The applied traction on the crack face, in order to eliminate the incident wave as indicated in (18), has the functional form $\mathrm{e}^{s \lambda x}$. Since the solutions for applying traction $\mathrm{e}^{s \eta x}$ to crack faces have been obtained in Section 2, the reflected and diffracted field can be constructed by superimposing the incident wave traction that is equal and opposite to (18). When we combine (7) and (18), the solution for $\bar{w}^{A}$ for $A$ wave (the first wave diffracted from the crack tip $A$ ) can be expressed as follows

$$
\begin{aligned}
\bar{w}^{A}(x, y, s)= & \frac{-1}{2 \pi i} \int_{\Gamma_{\eta_{1}}} p \mathrm{e}^{-s\left(\alpha h_{2}+\eta_{1} h_{1}\right)} \frac{1}{2 \pi i} \\
& \times \int_{\Gamma_{\eta_{2}}} \frac{-\mathrm{e}^{-s \alpha y+s \eta_{2} x}}{\mu s \alpha_{+}\left(\eta_{1}\right)\left(\eta_{1}-\eta_{2}\right) \alpha_{-}\left(\eta_{2}\right)} \mathrm{d} \eta_{2} \mathrm{~d} \eta_{1}, \\
= & \frac{1}{(2 \pi i)^{2}} \int_{\Gamma_{\eta_{1}}} \int_{\Gamma_{\eta_{2}}} \frac{p \mathrm{e}^{-s\left(\alpha h_{2}+\eta_{1} h_{1}\right)} \mathrm{e}^{-s \alpha y+s \eta_{2} x}}{\mu s \alpha_{+}\left(\eta_{1}\right)\left(\eta_{1}-\eta_{2}\right) \alpha_{-}\left(\eta_{2}\right)} \mathrm{d} \eta_{2} \mathrm{~d} \eta_{1} .
\end{aligned}
$$

The corresponding stress intensity factor expressed in the Laplace transform domain is

$$
\bar{K}^{d 1}(s)=\bar{K}^{A}(s)=\frac{1}{2 \pi i} \int_{\Gamma_{\lambda}} \frac{\sqrt{2} p \mathrm{e}^{-s\left(\alpha h_{2}+\lambda h_{1}\right)}}{\sqrt{s} \alpha_{+}(\lambda)} \mathrm{d} \lambda,
$$

where the superscript $d 1$ indicates that the first wave affects the right edge of the crack.

The dynamic stress intensity factor at the crack tip $A$ induced by the incident $\bar{\tau}_{y z}^{i}$ wave expressed in time domain will be

$$
K^{d 1}(t)=K^{A}(t)=p \sqrt{\frac{2}{\pi r_{A}}} \sin \left(\frac{\theta_{A}}{2}\right) H\left(t-b r_{A}\right)
$$


where

$$
r_{A}=\left(h_{1}^{2}+h_{2}^{2}\right)^{1 / 2}, \quad \theta_{A}=\cos ^{-1}\left(\frac{h_{1}}{r_{A}}\right) .
$$

The result expressed in (21) is the well-known solution for the dynamic stress intensity factor for a semi-infinite crack in an unbounded medium and subjected to a dynamic body force. It is interesting to note that the dynamic stress intensity factor jumps from zero to the corresponding static value after the incident cylindrical wave generated from the loading point arrived at the crack tip.

After the first incident wave arrived at the crack tip $A$, the second wave which passes $A$ is the one that is induced by the incident wave which arrives at the left tip $B$, and generates the diffracted $B$ wave, and then propagates towards $A$. If we follow the similar procedure that is used for constructing the $A$ wave, the $B$ wave can be constructed in the coordinate system $\left(x^{\prime}, y^{\prime}\right)$ by eqs.(17) and (7) as follows

$$
\begin{aligned}
& \bar{w}^{B}\left(x^{\prime}, y^{\prime}, s\right) \\
& \quad=\frac{-1}{(2 \pi i)^{2}} \int_{\Gamma_{\eta_{1}}} \int_{\Gamma_{\eta_{2}}} \frac{p \mathrm{e}^{-s\left[\alpha h_{2}-\eta_{1}\left(l+h_{1}\right)\right]} \mathrm{e}^{-s \alpha y^{\prime}+s \eta_{2} x^{\prime}}}{\mu s \alpha_{+}\left(\eta_{1}\right)\left(\eta_{1}-\eta_{2}\right) \alpha_{-}\left(\eta_{2}\right)} \mathrm{d} \eta_{2} \mathrm{~d} \eta_{1} .
\end{aligned}
$$

When the diffracted $B$ wave arrives at the right tip of the finite crack after some time, it carries with it a discontinuous displacement in the $z$-direction which violates the boundary condition for $x>0$. In order to satisfy the boundary condition where the displacement must be continuous for $x>0$, a distributed screw dislocation is required to close the crack opening displacement. The diffracted $B A$ wave will be induced when the $B$ wave arrives at the crack tip $A$. Now, if we change the $B$ wave from $\left(x^{\prime}, y^{\prime}\right)$ to $(x, y)$ coordinate system, then the displacement which must be eliminated ahead of the right tip $A$ is

$$
\begin{aligned}
& \bar{w}^{B}(x, y, s) \\
& \quad=\frac{1}{(2 \pi i)^{2}} \int_{\Gamma_{\eta_{1}}} \int_{\Gamma_{\eta_{2}}} \frac{p \mathrm{e}^{-s\left[\alpha h_{2}-\eta_{1}\left(l+h_{1}\right)\right]} \mathrm{e}^{-s \alpha y+s \eta_{2}(x+l)}}{\mu s \alpha_{+}\left(\eta_{1}\right)\left(\eta_{1}+\eta_{2}\right) \alpha_{-}\left(-\eta_{2}\right)} \mathrm{d} \eta_{2} \mathrm{~d} \eta_{1} .
\end{aligned}
$$

Again we treat the crack as a semi-infinite crack which lies along the line $y=0,-\infty<x<0$. The diffracted $B A$ wave can be obtained by superimposing the distributed dislocation that is equal and opposite to (23) ahead of the tip $x>0$ in the Laplace transform domain as follows

$$
\begin{aligned}
\bar{w}^{B A}(x, y, s)= & \frac{1}{(2 \pi i)^{2}} \int_{\Gamma_{\eta_{1}}} \int_{\Gamma_{\eta_{2}}} \frac{p \mathrm{e}^{-s\left[\alpha h_{2}-\eta_{1}\left(l+h_{1}\right)\right]} \mathrm{e}^{s \eta_{2} l}}{\mu s \alpha_{+}\left(\eta_{1}\right)\left(\eta_{1}+\eta_{2}\right) \alpha_{-}\left(-\eta_{2}\right)} \mathrm{d} \eta_{2} \mathrm{~d} \eta_{1} \\
& \times \frac{1}{2 \pi i} \int_{\Gamma_{\eta_{3}}} \frac{-\alpha_{-}\left(\eta_{2}\right) \mathrm{e}^{-s \alpha y+s \eta_{3} x}}{\left(\eta_{2}-\eta_{3}\right) \alpha_{-}\left(\eta_{3}\right)} \mathrm{d} \eta_{3} \\
= & \frac{1}{(2 \pi i)^{3}} \int_{\Gamma_{\eta_{1}}} \int_{\Gamma_{\eta_{2}}} \int_{\Gamma_{\eta_{3}}} \\
& \times \frac{p G\left(\eta_{1}, \eta_{2}\right) \mathrm{e}^{-s\left[\alpha h_{2}-\eta_{1}\left(l+h_{1}\right)\right]} \mathrm{e}^{s \eta_{2} l} \mathrm{e}^{-s \alpha y+s \eta_{3} x}}{\mu s \alpha_{+}\left(\eta_{1}\right)\left(\eta_{2}-\eta_{3}\right) \alpha_{-}\left(\eta_{3}\right)} \mathrm{d} \eta_{2} \mathrm{~d} \eta_{1} \mathrm{~d} \eta_{3},
\end{aligned}
$$


where

$$
G\left(\eta_{1}, \eta_{2}\right)=\frac{\alpha_{-}\left(\eta_{2}\right)}{\left(\eta_{1}+\eta_{2}\right) \alpha_{-}\left(-\eta_{2}\right)}
$$

Here (13) has been used to construct the solution for the diffracted $B A$ wave.

Using (23) and (14), the corresponding stress intensity factor expressed in the Laplace transform domain will be

$$
\begin{aligned}
\bar{K}^{d 2}(s)= & \bar{K}^{B A}(s) \\
= & \frac{1}{(2 \pi i)^{2}} \int_{\Gamma_{\eta_{1}}} \int_{\Gamma_{\eta_{2}}} \\
& \times \frac{\sqrt{2} p G\left(\eta_{1}, \eta_{2}\right) \mathrm{e}^{-s\left[\alpha h_{2}-\eta_{1}\left(l+h_{1}\right)\right]} \mathrm{e}^{s \eta_{2} l}}{\sqrt{s} \alpha_{+}\left(\eta_{1}\right)} \mathrm{d} \eta_{2} \mathrm{~d} \eta_{1} .
\end{aligned}
$$

The Cagniard-de Hoop method of Laplace inversion is used and the stress intensity factor in time domain is

$$
\begin{aligned}
K^{d 2}(t)= & K^{B A}(t) \\
= & \frac{-\sqrt{2} p}{2 \pi^{5 / 2}} \int_{b r_{B}+b l}^{t} \int_{b r_{B}}^{\tau-b l} \frac{1}{\sqrt{t-\tau}} \\
& \times \operatorname{Re}\left[\frac{G\left(\eta_{1}^{+}, \eta_{2}^{+}\right)}{\alpha_{+}\left(\eta_{1}^{+}\right)} \frac{\partial \eta_{1}^{+}}{\partial t_{1}} \frac{\partial \eta_{2}^{+}}{\partial t_{2}}-\frac{G\left(\eta_{1}^{-}, \eta_{2}^{+}\right)}{\alpha_{+}\left(\eta_{1}^{-}\right)} \frac{\partial \eta_{1}^{-}}{\partial t_{1}} \frac{\partial \eta_{2}^{+}}{\partial t_{2}}\right]_{t=\tau} \mathrm{d} t_{1} \mathrm{~d} \tau
\end{aligned}
$$

where

$$
\begin{aligned}
& \eta_{1}^{ \pm}=\frac{-t_{1} \cos \theta_{B}}{r_{B}} \pm \frac{i \sin \theta_{B}}{r_{B}}\left(t_{1}^{2}-b^{2} r_{B}^{2}\right)^{1 / 2}, \\
& \eta_{2}^{ \pm}=\frac{-t_{2}}{l} \pm i \varepsilon, \\
& r_{B}=\left[\left(l+h_{1}\right)^{2}+h_{2}^{2}\right]^{1 / 2}, \quad \theta_{B}=\cos ^{-1}\left(\frac{l+h_{1}}{r_{B}}\right), \quad t_{1}+t_{2}=t .
\end{aligned}
$$

Similarly, if we use the same process that is used for constructing the $B A$ wave, the diffracted $A B$ wave can be obtained from (19) and (13) and is expressed in the coordinate system of $\left(x^{\prime}, y^{\prime}\right)$ as follows

$$
\begin{aligned}
\bar{w}^{A B}\left(x^{\prime}, y^{\prime}, s\right) & \\
= & \frac{-1}{(2 \pi i)^{3}} \int_{\Gamma_{\eta_{1}}} \int_{\Gamma_{\eta_{2}}} \int_{\Gamma_{\eta_{3}}} \\
& \quad \times \frac{p G\left(\eta_{1}, \eta_{2}\right) \mathrm{e}^{-s\left(\alpha h_{2}+\eta_{1} h_{1}\right)} \mathrm{e}^{s \eta_{2} l} \mathrm{e}^{-s \alpha y^{\prime}+s \eta_{3} x^{\prime}}}{\mu s \alpha_{+}\left(\eta_{1}\right)\left(\eta_{2}-\eta_{3}\right) \alpha_{-}\left(\eta_{3}\right)} \mathrm{d} \eta_{3} \mathrm{~d} \eta_{2} \mathrm{~d} \eta_{1} .
\end{aligned}
$$

The $A B$ wave, which is generated by the diffracting $A$ wave at the left tip of the crack, will return back to the tip $A$ after some later time. After it arrives at the tip $A$, the boundary 
condition for $x>0$ will be violated again. As we did before, an appropriate sequence of screw dislocations along $x>0$ must be superimposed to close the opening displacement ahead of the crack tip. If we combine the result of (14) and (27), the corresponding stress intensity factor for tip $A$ in the Laplace transform domain can be obtained as follows

$$
\begin{aligned}
\bar{K}^{d 3}(s)= & \bar{K}^{A B A}(s) \\
= & \frac{1}{(2 \pi i)^{3}} \int_{\Gamma_{\eta_{1}}} \int_{\Gamma_{\eta_{2}}} \int_{\Gamma_{\eta_{3}}} \\
& \times \frac{\sqrt{2} p G\left(\eta_{1}, \eta_{2}\right) G\left(\eta_{2}, \eta_{3}\right) \mathrm{e}^{-s\left(\alpha h_{2}+\eta_{1} h\right)} \mathrm{e}^{s \eta_{2} l} \mathrm{e}^{s \eta_{3} l}}{\sqrt{s} \alpha_{+}\left(\eta_{1}\right)} \mathrm{d} \eta_{3} \mathrm{~d} \eta_{2} \mathrm{~d} \eta_{1} .
\end{aligned}
$$

Applying the inverse Laplace transform to (28), the stress intensity factor in time domain is

$$
\begin{aligned}
K^{d 3}(t)= & K^{A B A}(t) \\
= & \frac{-\sqrt{2} p}{2 \pi^{7 / 2}} \int_{b r_{A}+2 b l}^{t} \int_{b r_{A}}^{\tau-2 b l} \int_{b l}^{\tau-t_{1}-b l} \frac{1}{\sqrt{t-\tau}} \\
& \times \operatorname{Im}\left[\frac{G\left(\eta_{1}^{+}, \eta_{2}^{+}\right) G\left(\eta_{2}^{+}, \eta_{3}^{+}\right)}{\alpha_{+}\left(\eta_{1}^{+}\right)} \frac{\partial \eta_{1}^{+}}{\partial t_{1}} \frac{\partial \eta_{2}^{+}}{\partial t_{2}} \frac{\partial \eta_{3}^{+}}{\partial t_{3}}\right. \\
& \left.\quad-\frac{G\left(\eta_{1}^{-}, \eta_{2}^{+}\right) G\left(\eta_{2}^{+}, \eta_{3}^{+}\right)}{\alpha_{+}\left(\eta_{1}^{-}\right)} \frac{\partial \eta_{1}^{-}}{\partial t_{1}} \frac{\partial \eta_{2}^{+}}{\partial t_{2}} \frac{\partial \eta_{3}^{+}}{\partial t_{3}}\right]_{t=\tau} \mathrm{d} t_{2} \mathrm{~d} t_{1} \mathrm{~d} \tau
\end{aligned}
$$

where

$$
\begin{aligned}
& \eta_{1}^{ \pm}=\frac{t_{1} \cos \theta_{A}}{r_{A}} \pm \frac{i \sin \theta_{A}}{r_{A}}\left(t_{1}^{2}-b^{2} r_{A}^{2}\right)^{1 / 2}, \\
& \eta_{2}^{ \pm}=\frac{-t_{2}}{l} \pm i \varepsilon, \\
& \eta_{3}^{ \pm}=\frac{-t_{3}}{l} \pm i \varepsilon, \\
& t_{1}+t_{2}+t_{3}=t .
\end{aligned}
$$

For the time being, we have constructed in detail the first three waves which contribute the stress intensity factor at the right tip $A$ of the finite crack. Following a similar procedure, the complete solution for the dynamic stress intensity factor at tip $A$ that accounts for the contributions of all the diffracted waves induced from the two crack tips is finally obtained explicitly. The complete solution can be simplified into a very compact formulation as follows

$$
K(t)=\sum_{n=1}^{\infty} K^{d n}(t),
$$

where

$$
K^{d 1}(t)=p \sqrt{\frac{2}{\pi r_{A}}} \sin \left(\frac{\theta_{A}}{2}\right) \mathrm{H}\left(t-b r_{A}\right),
$$




$$
\begin{aligned}
& K^{d n}(t)=\frac{\sqrt{2} p(i)^{q}}{2 \sqrt{\pi} \pi^{n}(i)^{n}} \int_{b r_{1}+(n-1) b l}^{t} \int_{b r_{1}}^{a_{1}} \int_{b l}^{a_{2}} \int_{b l}^{a_{3}} \cdots \int_{b l}^{a_{n-1}} \\
& \quad \times \frac{1}{\sqrt{t-\tau}} \mathrm{Op}[\mathrm{BSIF}]_{t=\tau} \mathrm{d} t_{n-1} \mathrm{~d} t_{n-2}, \ldots, \mathrm{d} t_{1} \mathrm{~d} \tau, \text { for } n=2,3,4, \ldots,
\end{aligned}
$$

and

$$
\begin{aligned}
a_{1} & =\tau-(n-1) b l \\
a_{\nu} & =\tau-t_{1}-t_{2}-\cdots-t_{\nu-1}-(n-\nu) b l, \quad \nu=2,3,4, \ldots, n-1, \\
t_{1}+t_{2}+t_{3}+\cdots+t_{n}=t & \\
\mathrm{Op} & =\operatorname{Re}, \quad q=0, \quad \delta=-\left(l+h_{1}\right), \quad \text { when } n=2,4,6, \ldots, \\
\mathrm{Op} & =\mathrm{Im}, \quad q=1, \quad \delta=h_{1}, \quad \text { when } n=3,5,7, \ldots, \\
\mathrm{BSIF} & =\frac{G\left(\eta_{1}^{+}, \eta_{2}^{+}\right) G\left(\eta_{2}^{+}, \eta_{3}^{+}\right) \cdots G\left(\eta_{n-1}^{+}, \eta_{n}^{+}\right)}{\alpha_{+}\left(\eta_{1}^{+}\right)} \frac{\partial \eta_{1}^{+}}{\partial t_{1}} \frac{\partial \eta_{2}^{+}}{\partial t_{2}} \frac{\partial \eta_{3}^{+}}{\partial t_{3}} \cdots \frac{\partial \eta_{n}^{+}}{\partial t_{n}} \\
& -\frac{G\left(\eta_{1}^{-}, \eta_{2}^{+}\right) G\left(\eta_{2}^{+}, \eta_{3}^{+}\right) \cdots G\left(\eta_{n-1}^{+}, \eta_{n}^{+}\right)}{\alpha_{+}\left(\eta_{1}^{-}\right)} \frac{\partial \eta_{1}^{-}}{\partial t_{1}} \frac{\partial \eta_{2}^{+}}{\partial t_{2}} \frac{\partial \eta_{3}^{+}}{\partial t_{3}} \cdots \frac{\partial \eta_{n}^{+}}{\partial t_{n}},
\end{aligned}
$$

in which

$$
\begin{aligned}
& \eta_{1}^{ \pm}=\frac{-t_{1} \cos \theta_{1}}{r_{1}} \pm \frac{i \sin \theta_{1}}{r_{1}}\left(t_{1}^{2}-b^{2} r_{1}^{2}\right)^{1 / 2} \\
& \eta_{n}^{ \pm}=\frac{-t_{n}}{l} \pm i \varepsilon, \quad n=2,3,4, \ldots \\
& r_{1}=\left(\delta^{2}+h_{2}^{2}\right)^{1 / 2}, \quad \theta_{1}=\cos ^{-1}\left(\frac{-\delta}{r_{1}}\right) .
\end{aligned}
$$

The corresponding static solution for the stress intensity factor for this problem is

$$
K^{s 1}=2 p \sqrt{r_{B} /\left(2 \pi l r_{A}\right)} \sin \left(\frac{\theta_{A}-\theta_{B}}{2}\right) .
$$

In this section, the exact transient dynamic stress intensity factor for a finite crack subjected to anti-plane body forces has been derived. The induced wave fronts of incident and diffracted waves in a short time period are plotted in Figure 2. Numerical calculations have been done here only for the right tip of the crack. Figures 3-5 show the dimensionless stress intensity factors $K l^{1 / 2} / p$ for $r_{A}=0.25 l, 0.5 l$ and $3 l$ respectively, versus the dimensionless time $t / b r_{A}$ for various values of $\theta_{A}$. The corresponding time of the diffracted waves which arrive at the crack tip $A$ is indicated in the figures. It is indicated in these figures that the dynamic stress factor will reach a peak when the incident wave arrives at the crack tip, which is greater than the static value, and then oscillates near the static value after the first three waves have passed through the crack tip.

Next, the point loadings located at $\theta_{A}=30^{\circ}$ and $150^{\circ}$ for different values of $r_{A} / l$ are selected for investigation and the results are shown in Figures 6 and 7 . Here the dimensionless time is selected to be $t / b l$. It is worthy to indicate an interesting phenomenon revealed in these 
figures. With the increment of $r_{A} / l$, for constant $\theta_{A}$, the dynamic stress intensity factors will oscillate around values larger than the ones of the correspond static solutions. In these cases, the elastodynamic overshot of the stress intensity factors becomes very significant. This is very important for the safety consideration in mechanical design.

The maximum dynamic overshot always happens when the incident wave generated from the body force reaches the crack tip, and remains constant before the diffracted wave generated from the other crack tip arrives. This solution corresponds to a semi-infinite crack subjected to a dynamic body force and is shown in (21). After the first diffracted wave passes the crack tip, the stress intensity factors start to decrease and oscillate near the corresponding static values. The ratio of the value for maximum overshot to the static value can be expressed as follows

$$
K^{\max }(t) / K^{s 1}=\sqrt{l / r_{B}} \frac{\sin \left(\theta_{A} / 2\right)}{\sin \left(\theta_{A} / 2-\theta_{B} / 2\right)} .
$$

\section{Stress intensity factor for applying point loading on the crack faces}

In this section, a similar problem for a finite crack subjected to a pair of anti-plane dynamic point loadings on crack faces at a finite distance $h$ from the right tip is investigated. The mathematical analysis for this problem mainly follows the method provided in the previous section. Consider a stress-free infinite elastic homogeneous medium containing a crack of length $l$. At $t=0$, a concentrated dynamic loading of magnitude $P$ acts at $x=-h(h<1)$ on each face of the crack as shown in Figure 8. Following the similar analytical procedure as indicated in the previous section, the complete solution for the dynamic stress intensity factor at the right tip $A$ can be simplified into a very compact form as follows

$$
K(t)=\sum_{n=1}^{\infty} K^{d n}(t)
$$

where

$$
\begin{aligned}
K^{d 1}(t)= & p \sqrt{\frac{2}{\pi h}} \mathrm{H}(t-b h), \\
K^{d n}(t)= & \frac{(-1)^{n-1} \sqrt{2 \delta} p}{\sqrt{\pi} \pi^{n}} \int_{b \delta+(n-1) b l}^{t} \int_{b \delta}^{a_{1}} \int_{b l}^{a_{2}} \int_{b l}^{a_{3}} \cdots \int_{b l}^{a_{n-1}} \\
& \times \frac{1}{\sqrt{t-\tau}} \operatorname{CSIF} \mathrm{d} t_{n-1} \mathrm{~d} t_{n-2} \ldots \mathrm{d} t_{1} \mathrm{~d} \tau, \text { for } n=2,3,4, \ldots,
\end{aligned}
$$

and

$$
\begin{gathered}
\operatorname{CSIF=}\left[\frac{\sqrt{t_{2}+b l} \sqrt{t_{3}+b l} \cdots \sqrt{t_{n}+b l}}{\sqrt{t_{1}-b \delta}\left(l t_{1}+\delta t_{2}\right)\left(t_{2}+t_{3}\right)\left(t_{3}+t_{4}\right) \cdots\left(t_{n-1}+t_{n}\right)}\right. \\
\frac{\sqrt{t_{2}-b l} \sqrt{t_{3}-b l} \cdots \sqrt{t_{n}-b l}}{t_{t=\tau}},
\end{gathered}
$$




$$
\begin{aligned}
& a_{1}=\tau-(n-1) b l, \\
& a_{\nu}=\tau-t_{1}-t_{2}-\cdots-t_{\nu-1}-(n-\nu) b l, \quad \nu=2,3,4, \ldots, n-1, \\
& \delta=h, \quad \text { when } n=3,5,7, \ldots, \\
& \delta=l-h, \quad \text { when } n=2,4,6, \ldots, \\
& t_{1}+t_{2}+t_{3}+\cdots+t_{n}=t .
\end{aligned}
$$

The associated wave fronts for a short time period are shown in Figure 9. The corresponding static solution for this case will be

$$
K^{s 2}=p \sqrt{\frac{2}{\pi}\left(\frac{1}{h}-\frac{1}{l}\right)} .
$$

The non-dimensional stress intensity factors $K / K^{s 2}$ at the right tip versus the non-dimensional time $t / b l$ for various values of $h / l$ are shown in Figure 10. It shows that the dynamic stress intensity factors oscillate around the respective static values after the third wave has passed through the right tip of the finite crack. Moreover, we can see that the further away the loading from the right tip $A$, the larger the dynamic overshot will be. This is a very important characteristic feature that must be taken into account for the dynamic fracture analysis. It is also very interesting to note that the maximum dynamic overshot always occurs when the incident wave arrives at the crack tip, which is just the solution for a semi-infinite crack subjected to dynamic loading on crack faces. The ratio of the value for maximum overshot to the static value can be written as follows

$$
K^{\max }(t) / K^{s 2}=\sqrt{\frac{1}{1-h / l}} .
$$

As shown in Figure 10, the ratio of the dynamic overshot will be $2, \sqrt{2} 2 / \sqrt{3}$ for $h / l=$ $0.75,0.5$ and 0.25 , respectively.

So far, the solution for the dynamic stress intensity factor of a finite crack subjected to a pair of concentrated loadings on the crack faces has been obtained in the previous discussion. It is noted that the solutions for more general crack face loading can be constructed on the basis of the result shown in (33). Suppose that a traction distribution $-p f(x)$ is suddenly applied over the interval $x_{1}<x<x_{2}$ to crack faces, then the dynamic stress intensity factor of the problem is

$$
K^{f}(t)=\int_{x_{1}}^{x_{2}} K(t ; x) f(x) \mathrm{d} x
$$

The solution to the problem of a finite crack subjected to a uniformly distributed dynamic loading on its faces $(-l<x<0)$ can be obtained by using (36). The numerical result is shown in Figure 11 in which $K^{s}$ is the corresponding static value. Sih and Chen [28] also investigated the same problem and their result is presented in Figure 11 for comparison. It can be seen that the stress intensity factor of our result will reach a higher peak than that in [28]. Achenbach [21] has studied the problem of a finite crack subjected to an incident horizontally polarized shear wave. He obtained the first wave influence on the stress intensity factor and concluded that the ratio of the value for maximum dynamic overshot to the corresponding 
static value will always be $4 / \pi$. It is shown in Figure 11 that the peak of the stress intensity factor for our result is just equal to $4 / \pi$. However, the result of Sih and Chen [28] reaches a peak lower than $4 / \pi$. Moreover, our result indicates that the stress intensity factor will oscillate very near the static value after the first three waves have passed the crack tip.

\section{Conclusions}

Because of the difficulties in mathematical complexity, analytical solutions for an elastic solid containing a finite crack subjected to dynamic loading are very rare. In conventional studies of a semi-infinite crack in an unbounded medium subjected to dynamic loading, the complete solution can be obtained by applying direct integral transform methods. If a dynamic cracked problem has a characteristic length, or if the loading condition is unsymmetrical, then the same procedure cannot be applied. In this study, we used a powerful superposition methodology, which is performed in the Laplace transform domain, and successfully applied it to solving the transient response of a finite crack contained in an unbounded medium. The material is loaded by a dynamic anti-plane body force and a pair of concentrated forces with Heaviside-function time dependence. Two useful fundamental solutions are proposed to solve this problem. The complete closed-form solution for the transient stress intensity factor is obtained and is expressed in a simple and compact formulation. Every term in the solution has its own physical meaning. The solutions are valid for an infinite length of time and have accounted for the contributions coming from all diffracted waves which propagate back and forth between the two tips.

Numerical calculations have been made for the two problems. The numerical result shows that the dynamic stress intensity factor will reach a maximum dynamic overshot when the incident wave arrives at the tip, and will then oscillate near the static value after the first three waves have passed through the crack tip. The ratio of the value for the maximum dynamic overshot to the static value is also presented. The results obtained in this investigation provide information that is very important to the study of dynamic fracture.

The solution obtained in this paper can be considered to be a Green function for the associated problem. The solutions to problems of any arbitrary spatially distributed loading, or more general time dependence, can be obtained by superposition. Furthermore, it is easy to extend the method proposed in this study to solve more complicated problems which involve interaction of a finite crack with boundaries.

\section{Acknowledgments}

The authors gratefully acknowledge the financial support of this research by the National Science Council (Republic of China) under Grant NSC 83-0401-E-002-115.

\section{References}

1. B. Noble, The Wiener-Hopf Technique, Pergamon Press (1958).

2. A.T. de Hoop, Representation theorems for the displacement in an elastic solid and their application to elastodynamic diffraction theory, Doctoral dissertation, Technische Hogeschool, Delft (1958).

3. J.F. Loeber and G.C. Sih, Diffraction of anti-plane shear waves by a finite crack. Journal of the Acoustical Society of America 44 (1968) 90-98.

4. G.C. Sih and J.F. Loeber, Wave propagation in an elastic solid with a line of discontinuity or finite crack. Quarterly of Applied Mathematics 27 (1969) 193-213.

5. S.A. Thau and T.H. Lu, Transient stress intensity factor for a finite crack in an elastic solid caused by a dilatational wave. International Journal of Solids and Structures 7 (1971) 731-750. 
6. B.V. Kostrov, Self-similar problems of propagation of shear cracks. Applied Mathematics and Mechanics (PMM) 28 (1964) 1077-1087.

7. L.M. Flitman, Waves generated by sudden crack in a continuous elastic medium. Applied Mathematics and Mechanics (PMM) 27 (1963) 938-953.

8. G.C. Sih and G.T. Embley, Impact response of a finite crack in plane extension. International Journal of Solids and Structures 8 (1972) 977-993.

9. E.P. Chen, Impact response of a finite crack in a finite strip under anti-plane shear. Engineering Fracture Mechanics 9 (1977) 719-724.

10. E.P. Chen, Sudden appearance of a crack in a stretched finite strip. Journal of Applied Mechanics 45 (1978) 277-280.

11. S. Itou, Transient response of a finite crack in a strip with stress-free edges. Journal of Applied Mechanics 47 (1980) 801-805.

12. S. Itou, Transient response of a finite crack in a half plane under impact load. Journal of Applied Mechanics 48 (1981) 534-538.

13. L.B. Freund, The stress intensity factor due to normal impact loading of the faces of a crack. International Journal of Engineering Science 12 (1974) 179-189.

14. L.M. Brock, Shear and normal impact loading on one face of a narrow slit. International Journal of Solids and Structures 18 (1982) 467-477.

15. L.M. Brock, Stresses in a surface obstacle undercut due to rapid indentation. Journal of Elasticity 14 (1984) $415-424$.

16. L.M. Brock, M. Jolles and M. Schroedl, Dynamic impact over a subsurface crack: applications to the dynamic tear test. Journal of Applied Mechanics 52 (1985) 287-290.

17. C.C. Ma and Y.C. Hou, Theoretical analysis of the transient response for a stationary in-plane crack subjected to dynamic impact loading. International Journal of Engineering Science 28 (1990) 1321-1329.

18. C.C. Ma and Y.C. Hou, Transient analysis for anti-plane crack subjected to dynamic loadings. Journal of Applied Mechanics 58 (1991) 703-709.

19. L.B. Freund, Dynamic Fracture Mechanic, Cambridge University Press, Cambridge (1990).

20. B.V. Kostrov, Unsteady propagation of longitudinal shear cracks, Applied Mathematics and Mechanics (PMM) 30 (1966) 1241-1248.

21. J.D. Achenbach, Brittle and ductile extension of a finite crack by a horizontally polarized shear wave. International Journal of Engineering Science 8 (1970) 947-966.

22. J.D. Achenbach, Extension of a crack by a shear wave. Z. Angew. Math. Phy. 21 (1970) 887-900.

23. L.M. Brock, Effects of secondary diffractions on the stress intensity factors generated for a finite crack by a shear wave. International Journal of Engineering Science 13 (1975) 851-859.

24. C.H. Tsai and C.C. Ma, Transient analysis of a semi-infinite crack subjected to dynamic concentrated forces. Journal of Applied Mechanics 59 (1992) 804-811.

25. C.C. Ma and S.K. Chen, Exact transient analysis of an anti-plane semi-infinite crack subjected to dynamic body forces. Wave Motion 17 (1993) 161-171.

26. C.H. Tsai and C.C. Ma, The stress intensity factor of a subsurface inclined crack subjected to dynamic impact loading. International Journal of Solids and Structures 30 (1993) 2163-2175.

27. C.C. Ma and S.K.' Chen, Exact transient full-field analysis of an anti-plane subsurface crack subjected to dynamic impact loading. Journal of Applied Mechanics 61 (1994) 649-655.

28. G.C. Sih and E.P. Chen, Mechanics of Fracture 6, Cracks in Composite Materials, Martinus Nijhoff Publishers, (1981). 